\title{
Ic-instanton exact solution
}

\section{Érica E. Leite* and Luiz A. Ferreira}

Instituto de Física Teórica - IFT

E-mail: 'erica@ift.unesp.br, laf@ift.unesp.bri

\section{Loriano Bonora}

SISSA

E-mail: 'bonora@he.sissa.itit'

\section{Clisthenis P. Constantinidis}

$U F E S$

E-mail: 'clisthen@verao.cce.ufes.brìn

Abstract: We have obtained an exact solution of a supersymmetric Yang-Mills theory, constructed with the aid of the algebraic Leznov-Saveliev method. A particular set of equations - namely the sinh-Gordon model ones - is achieved by looking for BPS states. The classical solutions, knowed as IC-Instantons, interpolates initial and final string configuration.

\section{Introduction}

The $\mathcal{N}=4$ supersymmetric Yang-Mills theory $(\mathcal{N}=4 \mathrm{SYM})$ in $4 d$ is well known by its self-duality properties. In their work, Bonora et. al. [1] theory, putting on evidence an unexplored non-perturbative sector based on a new type of instanton. These new type of solution is knowed as interaction-carrying instanton or IC-INSTANTON, and emerge outside the context of duality as BPS classical instantons that connect distinct closed string configurations. In the strong coupling regime, these solutions correspond to $2 d$ complex manifolds which asymptotically has boundaries represented by one dimensional manifolds. This furnish an interpretation in terms of string scattering. By the same way that the instantons represent solutions interpolating distinct vacua in a theory, the IC-INSTANTON connect initial and final scattering states of strings.

For what follows, we consider the theory described by the action

$$
\begin{aligned}
\mathcal{S}=\frac{1}{\pi} \int d^{2} w \operatorname{Tr} & \left(D_{w} X^{i} D_{\bar{w}} X^{i}-\frac{1}{4 g^{2}} F_{w \bar{w}}^{2}-\frac{g^{2}}{2}\left[X^{i}, X^{j}\right]^{2}\right. \\
& \left.+i\left(\theta_{s}^{-} D_{\bar{w}} \theta_{s}^{-}+\theta_{c}^{+} D_{w} \theta_{c}^{+}\right)+i g \theta^{T} \Gamma_{i}\left[X^{i}, \theta\right]\right)
\end{aligned}
$$

${ }^{*}$ Speaker. 
that came out from the compactification in a cylinder of a type IIA string theory in 10 dimensions (see [i] $\left[\begin{array}{l}{[1} \\ 1\end{array}\right)$. The euclidean version of this action is invariant under the the supersymmetric transformations

$$
\begin{aligned}
\delta X^{i} & =\frac{i}{g}\left(\epsilon_{s}^{-} \gamma^{i} \theta_{c}^{+}+\epsilon_{c}^{+} \tilde{\gamma}^{i} \theta_{s}^{-}\right) \\
\delta \theta_{s}^{-} & =\left(-\frac{i}{2 g^{2}} F_{w \bar{w}}+\frac{1}{2}\left[X^{i}, X^{j}\right] \gamma_{i j}\right) \epsilon_{s}^{-}-\frac{1}{g} D_{w} X^{i} \gamma_{i} \epsilon_{c}^{+} \\
\delta \theta_{c}^{+} & =\left(\frac{i}{2 g^{2}} F_{w \bar{w}}+\frac{1}{2}\left[X^{i}, X^{j}\right] \tilde{\gamma}_{i j}\right) \epsilon_{c}^{+}-\frac{1}{g} D_{\bar{w}} X^{i} \tilde{\gamma}_{i} \epsilon_{s}^{-} \\
\delta A_{w} & =-2 \epsilon_{s}^{-} \theta_{s}^{-}, \quad \delta A_{\bar{w}}=-2 \epsilon_{c}^{+} \theta_{c}^{+}
\end{aligned}
$$

The BPS IC-INSTANTONS appears as classical solutions that preserves half of supersymmetry, what means that we search for solutions that satisfy the conditions

$$
\begin{array}{rlrl}
\left(\frac{i}{2 g^{2}} F_{w \bar{w}}+\frac{1}{2}\left[X^{i}, X^{j}\right] \tilde{\gamma}_{i j}\right) \epsilon_{c}^{+} & =0 & D_{w} X^{i} \gamma_{i} \epsilon_{c}^{+}=0 \\
\left(-\frac{i}{2 g^{2}} F_{w \bar{w}}+\frac{1}{2}\left[X^{i}, X^{j}\right] \gamma_{i j}\right) \epsilon_{s}^{-}=0 & D_{\bar{w}} X^{i} \tilde{\gamma}_{i} \epsilon_{s}^{-}=0
\end{array}
$$

After some manipulations, taking $X^{i}=0$ for all $i$ except two of them (suppose $X^{i} \neq 0$ for $i=1,2$ ), and defining $X \equiv X^{1}+i X^{2}$, the equations of motion of the theory take the form

$$
\begin{aligned}
F_{w \bar{w}}+i g^{2}[X, \bar{X}] & =0 \\
D_{w} X=0, \quad D_{\bar{w}} \bar{X} & =0
\end{aligned}
$$

We can also think of these equations as a reduction to two dimensions of the self duality conditions in four dimension. For instance, the self duality equations in $4 d$ are

$$
F_{y \bar{y}}+F_{z \bar{z}}=0 \quad F_{y z}=0=F_{\bar{y} \bar{z}}
$$

Imposing that nothing depends on the extra dimensions, i.e. $\partial_{z}=0=\partial_{\bar{z}}$, and making the identification $A_{z} \equiv X A_{\bar{z}} \equiv \bar{X}$ we get the same system as in $\overline{1} \cdot \overline{2}$ and $1 \overline{1} . \overline{3}$

From a mathematical point of view, 1' on a cylinder (or in a sphere with two punctures). The solutions of 1 parts: a branched covering of the cylinder through the characteristic polynomial relative to $X$; and a pure group factor that contains the coupling constant $g$.

\section{Two dimensional case}

We consider the two dimensional case of theory $1.1,1$, where we take $U(2)(N=2)$ as gauge group. We look for a pair $(A, X)$ - gauge potential and branched covering - that satisfies 1.1.1. In order to do this we take the ansatz

$$
X=Y^{-1} M Y ; \quad A_{w}=\partial_{w} Y^{\dagger}\left(Y^{-1}\right)^{\dagger} .
$$


In this ansatz, we parametrize $(\bar{M}) M$ with (anti)-holomorfic functions $(\bar{a}(\bar{w}))-a(w)$, which furnish a map for the covering; and the group element $Y$ is parametrized by a scalar field $\varphi$. More specifically

$$
M=\left(\begin{array}{ll}
0 & a \\
1 & 0
\end{array}\right) \quad Y=\left(\begin{array}{cc}
e^{\frac{\varphi}{2}} & 0 \\
0 & e^{-\frac{\varphi}{2}}
\end{array}\right)
$$

This made possible to translate the equations $1.12 .2,1.31$ into

$$
\partial_{w} \partial_{\bar{w}} \varphi-g^{2}\left(e^{2 \varphi}-|a| e^{-2 \varphi}\right)=0 .
$$

We can as well work with a field $u$, related to $\varphi$ by

$$
\varphi=u+\frac{1}{2} \ln |a|
$$

So, for this field, the equation $2, \overline{3}$ reads

$$
\partial_{w} \partial_{\bar{w}} u+\frac{1}{2} \partial_{w} \partial_{\bar{w}} \ln |a|=g^{2}|a| \sinh 2 u
$$

From now on we refer to this equation as the IC-INSTANTON equation.

\section{General Solution}

Since we are working with the two dimensional case, it is possible to use the algebraic Leznov-Saveliev method in order to construct explicitly the solutions. This method is based on the existence of an infinite dimensional Lie algebra, the Kac-Moody algebra, from where it emerges an infinite number of conserved quantities.

In the two dimensional case, the relevant potentials for describe the Hitchin system

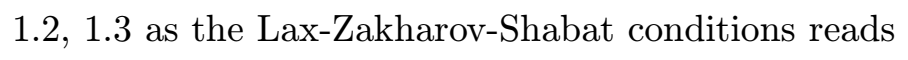

$$
A_{w} \equiv-\partial_{w} \gamma \gamma^{-1}+E_{-1} \quad A_{\bar{w}} \equiv \gamma E_{1} \gamma^{-1}
$$

where $\gamma \equiv e^{\varphi H^{0}}$ and

$$
E_{1} \equiv g T_{+}^{0}+g \bar{a}(\bar{z}) T_{-}^{1} \quad E_{-1} \equiv g a(z) T_{+}^{-1}+g T_{-}^{0}
$$

$T_{ \pm}^{n}$ are the generators of a Kac-Moody algebra $\widehat{s l}(2, \mathbb{C})$. By this way, the zero curvature conditions

$$
F_{\mu \nu}=0
$$

imply the equations of motion that, translated in terms of the field contents of the model assumes the form

$$
\partial_{w} \partial_{\bar{w}} \varphi=g^{2}\left(e^{2 \varphi}-|a|^{2} e^{-2 \varphi}\right)
$$

These corresponds exactly to $2 \overline{2} . \overline{3}$ ( or $2 \overline{2} . \overline{5}$ ). 
As a consequence of 3 , we can parametrized the connection by a group element

$$
A_{\mu} \equiv-\partial_{\mu} W W^{-1}
$$

With this, the integration independs on the path; so we can decompose $W$ using different group elements. In particular, taking two elements $g_{1}, g_{2}$ we can write

$$
W=g_{1}=\gamma g_{2} \quad \rightarrow \quad \gamma=g_{1} g_{2}^{-1}
$$

It follows from the method that

$$
g_{1}^{-1}|\lambda\rangle \equiv \text { function of } w \quad\langle\lambda| g_{2} \equiv \text { function of } \bar{w} .
$$

Here, $|\lambda\rangle$ are highest weight states of a $\widehat{s l}(2, \mathbb{C})$ representation. Then, from 3.6. that

$$
\left\langle\lambda\left|\gamma^{-1}\right| \lambda\right\rangle=\left\langle\lambda\left|g_{2} g_{1}^{-1}\right| \lambda\right\rangle
$$

Next, we use a Gauss decomposition associated to the principal grading [2] two group elements as

$$
g_{1} \equiv N \gamma_{-} M_{-} \quad g_{2} \equiv M \gamma_{+} N_{+}
$$

The indices refers to the degree of operators that enters in the exponentiation of element, that is, $X_{+}$only contains operators of positive degree with respect to the grading, $X_{+}=$ $e^{\mathcal{G}_{+}}$. In particular,

$$
\gamma_{+}=e^{\theta_{+}(\bar{w}) H^{0}}, \quad \gamma_{-}=e^{\theta_{-}(w) H^{0}}
$$

This allow us to write $3 . \overline{8}$ as

$$
\left\langle\lambda\left|\gamma^{-1}\right| \lambda\right\rangle=\left\langle\lambda\left|\gamma_{+}\left(x_{+}\right) N_{+}\left(x_{+}\right) M_{-}^{-1}\left(x_{-}\right) \gamma_{-}^{-1}\left(x_{-}\right)\right| \lambda\right\rangle
$$

Except for $\gamma_{ \pm}$, the parameters $N_{+}$and $M_{-}$are determined by relations encoded in "3.6.".

With these ingredients we are able to write the general solution to the model. For this, two distinct maximal weight representations of $\widehat{s l}(2, \mathbb{C})$ are needed:

$$
e^{-\varphi}=\frac{\left\langle\lambda_{1}\left|N_{+}\left(x_{+}\right) M_{-}^{-1}\left(x_{-}\right)\right| \lambda_{1}\right\rangle}{\left\langle\lambda_{0}\left|N_{+}\left(x_{+}\right) M_{-}^{-1}\left(x_{-}\right)\right| \lambda_{0}\right\rangle} e^{\theta_{+}-\theta_{-}}
$$

So, the general solution of depends on the parameters $\theta_{ \pm}$, and on the elements $N_{+}, M_{-}$. This group elements are determined by

$$
\begin{gathered}
\partial_{\bar{w}} N_{+} N_{+}^{-1}=-g\left(e^{-2 \theta_{+}} T_{+}^{0}+\bar{a}(\bar{w}) e^{2 \theta_{+}} T_{-}^{1}\right) \\
\partial_{w} M_{-} M_{-}^{-1}=-g\left(a(w) e^{-2 \theta_{-}} T_{+}^{-1}+e^{2 \theta_{-}} T_{-}^{0}\right)
\end{gathered}
$$

The $\theta_{ \pm}$functions will be fix by imposing the boundary conditions, as we will see next. 


\section{Boundary Conditions}

We can easily understand how to select a particular solution outside the general solution making a change of variable. Let

$$
\frac{d \zeta}{d w}=\sqrt{a} \quad \frac{d \bar{\zeta}}{d \bar{w}}=\sqrt{\bar{a}}
$$

We note that, in terms of this new variable, the IC-INSTANTON equation $2.51(3.4)$ is equivalent to that of the sinh-Gordon model with a source

$$
\partial_{\zeta} \partial_{\bar{\zeta}} u-2 g^{2} \sinh 2 u=-\frac{\pi}{4} \delta_{a}(a, \bar{a})\left(\partial_{\zeta} a\right)\left(\partial_{\bar{\zeta}} \bar{a}\right)
$$

The source has the effect of impose boundary conditions; in other words, the solutions $u$ satisfies the sinh-Gordon homogeneous equations together with the boundary conditions

$$
u \sim-\frac{1}{2} \log |a| \quad \varphi \sim \text { finite } \quad a \sim 0
$$

The solution $u$ diverges logarithmically at the zeros of $a$, implying that $\varphi$ must be finite at the same point. Apart this, far from the zeros of $a$ we have

$$
u \sim \text { finite } \quad \varphi \sim \frac{1}{2} \log |a| \quad a \sim \infty
$$

\subsection{The $\zeta$ variable}

Here, it should be appropriate to do a comment about the role of the branched covering $M$. We must have this in mind when dealing with the changes of variable performed and, in particular, with the equation 4.2. . More details can be found in [6]

The matrix $M$ represents a branched covering of the cylinder spanned by the coordinate $w$. It is convenient now to pass to a new coordinate $z=e^{w}$, which maps the cylinder into the complex $z$-plane with two punctures at $z=0$ and $z=\infty$. The eigenvalues of $M$, which are the roots of the algebraic equation $X^{2}=a$ can be thought of as the sheets of a double covering of the cylinder. Each sheet is a copy of the complex $z$-plane, so, each eigenvalue spans a sheet. The points where the eigenvalues coincide are the branched points. Let us consider the equation for an hyper-elliptic Riemann surface $\Sigma$

$$
y^{2}=a(z)=\left(z-z_{1}\right)\left(z-z_{2}\right) \ldots\left(z-z_{n}\right)
$$

There are branch points at $z=z_{1}, \ldots, z=z_{n} . y$ and $z$ are coordinates of two complex planes, but, of course they can be considered as function over $\Sigma$. The coordinate $z$ is not a good coordinate near a branch point. A good local coordinate near a branch point $z_{i}$ is $\xi_{i}=\sqrt{z-z_{i}}$. I.e., near $z_{i}$ we have $z=z_{i}+\xi_{i}{ }^{2}$.

Since near a branch point, neither $y$ or $z$ are good coordinates, the usual delta function is given by $\delta(\xi, \bar{\xi})$. But, after some considerations, we see that we have the relations

$$
\delta_{a}(a, \bar{a}) \sim 2 \delta(\xi, \bar{\xi}), \quad \delta_{\zeta}(\zeta, \bar{\zeta}) \sim 3 \delta(\xi, \bar{\xi})
$$


This provide us with a way of transform locally the delta $\delta_{a}(a, \bar{a})$ in a function of $\zeta$ variable, using 14.6 . We must take into account the Jacobian factor due to the change of coordinates, what lead us to the relation

$$
\delta_{\zeta}(\zeta, \bar{\zeta})=\frac{3}{2} \delta_{a}(a, \bar{a})\left(\partial_{\zeta} a\right)\left(\partial_{\bar{\zeta}} \bar{a}\right)
$$

We return to $\underline{4} \cdot \overline{4} \cdot \overline{1}$ and get

$$
\partial_{\zeta} \partial_{\bar{\zeta}} u-2 g^{2} \sinh 2 u=-\frac{\pi}{6} \delta_{\zeta}(\zeta, \bar{\zeta})
$$

This means that near a branch point, via the local coordinate $\xi$, we can solve the equation for $u$ as a function of $\zeta$; the $a$ dependence is restored via 4 . We will in this way obtain some sort of "partials" solutions, localized around a branched point, that we must be able to patch together in order to it spreads out globally. Actually, we did not yet completely understood the way of gluing this regions.

\section{Choice of $\theta$}

Now we have the necessary elements to fix the $\theta$ parameters:

$$
\theta_{+}=-\frac{1}{4} \ln \bar{a} \quad \theta_{-}=\frac{1}{4} \ln a
$$

We can substitute this expression in $2 . \overline{4}, \overline{3} \cdot 1$ to get

$$
e^{-u}=\frac{\left\langle\lambda_{1}\left|N_{+} M_{-}^{-1}\right| \lambda_{1}\right\rangle}{\left\langle\lambda_{0}\left|N_{+} M_{-}^{-1}\right| \lambda_{0}\right\rangle}
$$

This choice of $\theta$ also simplifies the integration of elements $N_{+}, M_{-}$. Indeed, becomes

$$
\partial_{\bar{w}} N_{+} N_{+}^{-1}=-g \sqrt{\bar{a}(\bar{w})} b_{1} \quad \partial_{w} M_{-} M_{-}^{-1}=-g \sqrt{a(w)} b_{-1} .
$$

The operators $b_{1}$ and $b_{-1}$ are elements of a Heisenberg sub-algebra of the $\widehat{s l}(2)$ Kac-Moody algebra. That is like an algebra of harmonic oscillators, i.e. they are generated by

$$
b_{2 n+1} \equiv T_{+}^{n}+T_{-}^{n+1} \quad\left[b_{2 m+1}, b_{2 n+1}\right]=C(2 m+1) \delta_{m+n+1,0}
$$

We can then integrate

$$
\begin{array}{lr}
N_{+}=e^{I_{+} b_{1}} h_{+} & I_{+}=-g \int d \bar{w} \sqrt{\bar{a}(\bar{w})} \\
M_{-}=e^{I_{-} b_{-1}} h_{-} & I_{-}=-g \int d w \sqrt{a(w)}
\end{array}
$$




\subsection{IC-INSTANTON insight}

We come to a crucial point, that will permit us to point out the exact expression for the IC-INSTAnton. The Leznov-Saveliev method provide us the general expression for the solution. The boundary conditions fixes the parameters of a particular class of solutions. At this stage, according to $5.2,5.5,5.6$, we must solve

$$
\left\langle\lambda\left|N_{+} M_{-}^{-1}\right| \lambda\right\rangle=\left\langle\lambda\left|\mathrm{g}(x) \mathbf{h ~ g}^{-1}(x)\right| \lambda\right\rangle
$$

where $\mathbf{h} \equiv h_{+} h_{-}^{-1}$ is the integration constant, and $\mathrm{g}(x)$ is the group element

$$
\mathrm{g}(x)=e^{I_{-} b_{-1}} e^{I_{+} b_{1}} .
$$

The peculiarity of IC-INSTANTON solution is the particular choice of the integration constant h. Usually, for the sinh-Gordon theory, the one soliton solution is obtained taking $\mathbf{h}$ to be the exponential of a vertex operator, $\mathbf{h}=e^{V(\mu)}$, where $V(\mu)$ is an element of the Kac-Moody algebra which is an eigenvectos of the adjoint action of the oscillators $b_{ \pm 1}$, i.e.,

$$
\left[b_{2 n+1}, V(\mu)\right]=-2 \mu^{2 n+1} V(\mu)
$$

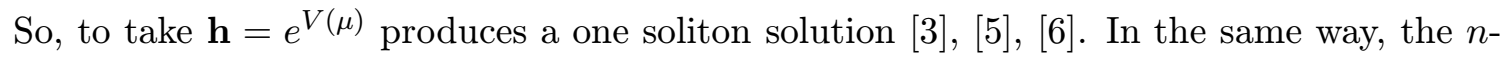
soliton solution is obtained by taking $\mathbf{h}$ as a product of those exponentials, $\mathbf{h}=\prod_{i=1}^{n} e^{V\left(\mu_{i}\right)}$. But here, to obtain the IC-INSTANTON solution we must take a continuous infinite product of those exponentials

$$
h_{+} h_{-}^{-1} \equiv \prod_{i=1}^{\infty} e^{V\left(\mu_{i}\right)}
$$

This means that we have an $N$-soliton solution, with $N \rightarrow \infty$; this is some sort of soliton condensate [4]

At this point, we determined all elements present in the construction of solution. Now, we have to do two more things: i) evaluate the expected value 5.5 for that appear in the expression for the solution for $N \rightarrow \infty$; ii) and take the continuous limit for the soliton condensate.

So, first, let us deal with the expected value 5.74 After this choice of the constant $\mathbf{h}$ we can rewrite

$$
\begin{aligned}
& \left\langle\lambda_{0}\left|N_{+} M_{-}^{-1}\right| \lambda_{0}\right\rangle=\operatorname{det}(1+\mathcal{W}) \\
& \left\langle\lambda_{1}\left|N_{+} M_{-}^{-1}\right| \lambda_{1}\right\rangle=\operatorname{det}(1-\mathcal{W})
\end{aligned}
$$

where $\mathcal{W}$ is the infinite matrix

$$
\mathcal{W}_{i j}=e^{\frac{\beta\left(\mu_{i}\right)}{2}} \frac{\sqrt{4 \mu_{i} \mu_{j}}}{\mu_{i}+\mu_{j}} e^{\frac{\beta\left(\mu_{j}\right)}{2}} .
$$

The $\beta(\mu)$ 's are functions of $I_{ \pm}$that appears in $5.5,5.6$ :

$$
\beta\left(\mu_{i}\right)=-2\left(\mu_{i} I_{+}+\frac{I_{+}}{\mu_{i}}\right)
$$


Due the considerations in section $4 . \overline{4}$, we are able to evaluate $I_{ \pm}$for regions around a zero of $a(w)$; that is, near the zeros of $a(w)$ we get

$$
\beta\left(\mu_{i}\right)=2 g\left(\mu_{i} \bar{\zeta}+\frac{\zeta}{\mu_{i}}\right) \quad \text { once that } \quad I_{+}=-g \bar{\zeta} ; I_{-}=-g \zeta \quad \text { as } \quad a \sim 0
$$

So, according to 5.21 and $5.11,5,12$, we get

$$
u=\ln \left(\frac{\operatorname{det}(1+\mathcal{W})}{\operatorname{det}(1-\mathcal{W})}\right)=\operatorname{Tr} \ln \frac{1+\mathcal{W}}{1-\mathcal{W}}
$$

This means that we then have an infinite series of powers of $\mathcal{W}$ matrix

$$
u=\sum_{n=0}^{\infty} \frac{\operatorname{Tr} \mathcal{W}^{2 n+1}}{2 n+1}
$$

We pass now to the second step, that is, take the continuous limit. This can be achieved by transforming the matrix indices of $\mathcal{W}$ into continuous ones; this means that, to take the trace we must perform integrations instead of summations. This passage has some subtle aspects, and we must take some care (see [6] for further details). In this procedure, we introduce a scaling factor $(\Lambda)$ in the integration measure, that have to be fixed correctly in order to satisfy the boundary conditions. We then pass from 5

$$
u=2 \sum_{n=0}^{\infty} \frac{(2 \Lambda)^{2 n+1}}{2 n+1} I_{2 n+1}
$$

In this expression, $I_{2 n+1}$ are the integrals

$$
I_{2 n+1}=\frac{1}{2^{2 n}} \int_{-\infty}^{\infty} \ldots \int \mathrm{d} \phi_{1} \ldots \mathrm{d} \phi_{2 n} \frac{K_{0}\left(4|g||\zeta| \sqrt{w_{2 n+1}(\phi)}\right)}{\cosh \left(\frac{\phi_{1}}{2}\right) \ldots \cosh \left(\frac{\phi_{2 n}}{2}\right) \cosh \left(\sum_{i=1}^{2 n} \frac{\phi_{i}}{2}\right)}
$$

where the variables $\phi$ 's are related to the $\mu$ 's, and the functions $w_{N}$ are

$$
w_{N}(\phi)=N+2 \sum_{l=0}^{N-2} \sum_{m=1}^{N-l-1} \cosh \sum_{n=m}^{m+l} \phi_{n}
$$

The solution $5 . \overline{18}$ with a free parameter $\Lambda$ satisfies the equation $\overline{4} .2$ in all regions, except near the singularities. In this region, we can expand the equation in terms of powers of $\Lambda$, what give us an infinite number of differential non-linear equations

$$
\begin{aligned}
I_{3}^{\prime \prime}+\frac{1}{x} I_{3}^{\prime}-I_{3} & =8 I_{1}^{3} \\
I_{5}^{\prime \prime}+\frac{1}{x} I_{5}^{\prime}-I_{5} & =\frac{40}{3} I_{1}^{2} I_{3}+\frac{32}{3} I_{1}^{5} \\
I_{7}^{\prime \prime}+\frac{1}{x} I_{7}^{\prime}-I_{7} & =\frac{224}{9} I_{1}^{4} I_{3}+\frac{56}{9} I_{1} I_{3}^{2}+\frac{56}{5} I_{1}^{2} I_{5}+\frac{256}{45} I_{1}^{7} \\
\vdots & \vdots
\end{aligned}
$$


These relations are verified, as we can see in $[\overline{7}]$. So, 5.18 is indeed a solution.

We analyze the behavior of 5.18 near the regions where we have to obey the boundary conditions. So, by imposing that 4 :

$$
\Lambda=\frac{1}{4 \pi} .
$$

Therefore from 5

$$
u=2 \sum_{n=0}^{\infty} \frac{1}{(2 \pi)^{2 n+1}} \frac{I_{2 n+1}}{2 n+1}
$$

Acknowledgements E.E. Leite would like to thank FAPESP for financial support.

\section{References}

[1] G. Bonelli, L. Bonora, S. Terna, A. Tomasiello hep-th 9912227; - Nucl. PhYs. B554 (1999) 103-135, hep-th 9901093; - PHYs. LETt. 435B (1998) 303-311, hep-th 9805071; - Nucl. Phys. B538 (1999) 100-116, hep-th 9807232; - - Phys. LeTt. 410B (1997) 142-150, hep-th 9705137; and references therein.

[2] V.G. Kac Infinite Dimensional Lie Algebras, Cambridge, (1990).

[3] D.I. Olive, J.W.R. Underwood and M.V. Saveliev, Phys. Letr. 311B (1993) 117-122, hep-th 9212123.

[4] O. Babelon and D. Bernard, Int. J. Mod. Phys. A8 (1993) 507, hep-th 9206003.

[5] L.A. Ferreira, J. Luis Miramontes and J. Sánchez-Guillén, J. Math. PhYs. 38 (1997) 882-901, hep-th 9606066.

[6] L. Bonora, C.P. Constantinidis, L.A. Ferreira and E.E. Leite Construction of exact Riemannian instanton solutions hep-th 0208175.

[7] B. Mc Coy, C. Tracy and T.T. Wu, J. Math. Phys. 18 (1977) 1058. 